Article

\title{
Statistical Optimization of Urinary Organic Acids Analysis by a Multi-Factorial Design of Experiment
}

\author{
Marco Pazzi ${ }^{1, *}$, Sara Colella ${ }^{2}$, Eugenio Alladio ${ }^{1,3}$, M. Paola Puccinelli ${ }^{4}$, Giulio Mengozzi ${ }^{4}(\mathbb{C}$ \\ and Claudio Medana ${ }^{2}$ (D) \\ 1 Dipartimento di Chimica, Università degli Studi di Torino, Via Pietro Giuria 7, I-10125 Turin, Italy; \\ eugenio.alladio@unito.it \\ 2 Dipartimento di Biotecnologie Molecolari e Scienze per la Salute, Università degli Studi di Torino, \\ I-10125 Turin, Italy; sara.colella181@edu.unito.it (S.C.); claudio.medana@unito.it (C.M.) \\ 3 Centro Regionale Antidoping e di Tossicologia “A. Bertinaria”, Reg Gonzole 10-1, I-10043 Turin, Italy \\ 4 SC di Biochimica Clinica, Città della Salute e della Scienza, Corso Bramante 88, I-10126 Torino, Italy; \\ mpuccinelli@cittadellasalute.to.it (M.P.P.); gmengozzi@cittadellasalute.to.it (G.M.) \\ * Correspondence: marco.pazzi@unito.it; Tel.: +39-01-1670-5255; Fax: +39-01-1670-5247
}

Received: 30 July 2020; Accepted: 26 August 2020; Published: 28 August 2020

\begin{abstract}
The analysis of urinary organic acids is useful for patients suspected to have inborn errors of metabolism known as organic acidurias. These diseases cause an accumulation of organic acids in body fluids and their abnormal excretion in urines. By means of chemometrics tools, such as principal component analysis and multiple linear regression, it was concluded that the conditions used in our laboratory are really the most suitable to achieve high yields of analytes.
\end{abstract}

Keywords: acylglycines; urine; doe; GC-MS; inborn errors of metabolism; organic acidurias

\section{Introduction}

Organic acids and acylglycines determination in urine samples is an extremely important tool for the diagnosis of inborn errors of metabolism (IEM) characterized by abnormal excretion of these substances and known as organic acidurias [1]. Organic acidurias consist of a very heterogeneous group of disorders, both phenotypically and genetically. They are caused by defects in genes responsible for the coding of enzymes or cofactors involved in crucial metabolic pathways [2]. To date, according to Villani et al. [3] more than 65 organic acidurias have been documented. Even if these disorders are individually very rare, the cumulative incidence is probably one out of 3000 live births [4]. They are found in all ethnic groups and can occur at every age, but most frequently their onset is in the first days or months of life. Some of them can be treated, with promising outcomes. For this reason it is really important to diagnose them early, in order to start the treatment before the most severe symptoms take place.

Since isovaleric aciduria was identified by Tanaka and Isselbacher [5] with GC-MS, this analytical technique is considered the method of choice for the diagnosis of organic acidurias [6]. Other methods of detection were proposed, such as capillary electrophoresis [7,8], electrospray ionization tandem mass spectrometry [9], and NMR [10]. All of them, though, have relevant drawbacks, such as inadequate sensitivity and repeatability and limitations to provide the broad profile of urinary metabolites and the standardization offered by GC-MS.

The most common analytical strategy of IEM laboratories consists of liquid-liquid extraction (LLE) of metabolites from urine samples, followed by trimethylsilyl derivatization and GC-MS analysis [11]. The obtained chromatographic profiles allow the identification and quantification of more than 250 organic acids and acylglycines, among which almost a hundred metabolites are useful for the diagnosis of organic acidurias $[12,13]$. 
Urinary organic acids can be aliphatic or aromatic, linear, cyclic or heterocyclic, saturated or unsaturated, hydroxyl-, keto-, mono-, di- or tricarboxylic acids. Endogenous organic acids are intermediate metabolites of the catabolism of the most important components of the cells, such as amino acids, lipids, biogenic amines, nucleic acids and steroids [14]. They can also arise from exogenous sources, like xenobiotics and dietary supplements, food additives, drugs and drug metabolism or bacterial metabolism [15]. Organic acids are polar compounds rapidly accumulated and excreted in the urine, more than in other biological fluids. Acylglycines are synthetized in the liver by the conjugation of glycine with acyl-coenzyme A ( $\mathrm{CoA})$ esters resulting from organic acids. This conjugation is a detoxification system used by the organism to prevent the accumulation of acyl-CoA esters in some IEM [16].

The aim of the present study was the systematic evaluation of the method used in our laboratory to analyze organic acids and acyglycines for organic acidurias diagnosis. We performed a multi-factorial experimental design to investigate the dependence of the analytes total yield on experimental factors.

A design of experiment (DoE) is used to obtain as many information as possible with the minimum number of experiments. It is a strategy which consents to maximize the efficiency of the experiments and to minimize waste and costs [17]. Firstly it is required to choose factors, i.e., the quantities that affect the response, secondly to define levels for each factor. The levels are chosen with the purpose to maximize the information about the factors. Then, the multivariate data obtained from the experiments are fitted into an empirical function, usually linear or quadratic with interaction terms. The calculated function can be used to provide further information about the system [17]. The DoE strategy is claimed to be better than the traditional "one-change-at-a-time" approach, since the traditional method is unlikely to discover the optimal conditions, especially when factors seem to be correlated, thus leading to perform more experiments than necessary [18]. In the present study, since it was impossible to exclude interactions between the factors, we have used a full-factorial design with four factors and two levels, to obtain the most robust interpretation of their effects, together with their reciprocal interactions, on the analytical yield of the organic acids and acylglycines.

The final purpose of this DoE was to maximize the areas of the chromatographic peaks and to optimize the method used in our laboratory. The results were expressed in terms of principal components and were interpreted using a multiple linear regression (MLR) model.

\section{Materials and Methods}

\subsection{Reagents}

The analysis of organic acids and acylglycines was performed using the following reagents: saline solution $(0.9 \% w / v \mathrm{NaCl})$ from Fresenius Kabi (Varese, Italy); n-heptane analytical grade from VWR Chemicals (Milano, Italy); sodium hydroxide, hydroxylamine hydrochloride, tropic acid (internal standard), ethyl acetate analytical grade, N,O-Bis(trimethylsilyl)trifluoroacetamide (BSTFA), trimethylchlorosilane (TMCS) from Sigma-Aldrich (Milano, Italy); 30\%. w/w hydrochloric acid ( $\mathrm{HCl}$ ) from Fluka Analitics (Milano, Italy). A 35\% w/v solution was made of sodium hydroxide and $1 \mathrm{M}$ hydroxylamine hydrochloride. The derivatization reagent was prepared by adding to BSTFA $1 \% v / v$ of TMCS.

\subsection{Analytical Method}

The determination of organic acids and acylglycine in urine samples was performed according to the 2008 guidelines of the "Società Italiana Studio Malattie Metaboliche Ereditarie" [7] as follows:

1. Sample creatinine was determined according to Jaffè method;

2. Urine samples were diluted with saline solution to obtain a concentration of $2 \mathrm{mM}$ of creatinine;

3. A total of $1 \mathrm{~mL}$ of the diluted samples was added with $100 \mu \mathrm{L}$ of internal standard $(1 \mathrm{M}$ tropic acid), $20 \mu \mathrm{L}$ of a solution of $35 \% w / v$ sodium hydroxide and $100 \mu \mathrm{L}$ of 1 M hydroxylamine. Samples were incubated at $60^{\circ} \mathrm{C}$ for $30 \mathrm{~min}$; 
4. A total of $50 \mu \mathrm{L}$ of $30 \% w / w$ hydrochloric acid and approx. $0.5 \mathrm{~g}$ of sodium chloride were added. Extraction was performed three times with $2 \mathrm{~mL}$ of ethyl acetate. The samples were vigorously vortexed for $1 \mathrm{~min}$ or shaken at $350 \mathrm{rpm}$ on an automatic shaker for $10 \mathrm{~min}$;

5. The combined extracts were dried at $40{ }^{\circ} \mathrm{C}$ in a water bath, under a gentle nitrogen flux;

6. A total of $50 \mu \mathrm{L}$ of BSTFA with $1 \%$ of TMCS were added to dried extracts and they are heated at $50{ }^{\circ} \mathrm{C}$ for $40 \mathrm{~min}$;

7. Samples were diluted with $500 \mu \mathrm{L}$ of $\mathrm{n}$-heptane.

The analysis was performed with a GC-MS (model 6890/5973, Agilent Technologies, Milan, Italy) equipped with an Electron Ionization (EI) source, $60 \mathrm{~m}$ cross-linked $5 \mathrm{MS}{ }^{\circledR} 0.25 \mathrm{~mm} \times 0.25 \mu \mathrm{m}$ capillary column was used (CPS Analitica s.r.l., Milan, Italy). Carrier gas employed was helium with a flow of $0.8 \mathrm{~mL} / \mathrm{min}$. The solvent delay was set at $14 \mathrm{~min}$. Injections of $1 \mu \mathrm{L}$ were performed in splitless mode at $285^{\circ} \mathrm{C}$. The GC oven temperature was initially set at $60^{\circ} \mathrm{C}$, raised at a rate of $5{ }^{\circ} \mathrm{C} / \mathrm{min}$ up to $155^{\circ} \mathrm{C}$, where it was maintained for $5 \mathrm{~min}$. Then it was raised again at a rate of $5^{\circ} \mathrm{C} / \mathrm{min}$ up to $200^{\circ} \mathrm{C}$, and then at a rate of $50^{\circ} \mathrm{C} / \mathrm{min}$ up to $290^{\circ} \mathrm{C}$, where it was maintained for $9 \mathrm{~min}$, for an overall run time of $49.5 \mathrm{~min}$. Temperatures were set at $250{ }^{\circ} \mathrm{C}$ for the ionization source, at $280{ }^{\circ} \mathrm{C}$ for the transfer line and at $180^{\circ} \mathrm{C}$ for the quadrupole. The spectra are acquired in scan mode, with a range of analysis between 60 and $500 \mathrm{~m} / \mathrm{z}$.

\subsection{Urine Specimens}

Urine samples used for this study were control materials ORG ${ }^{\circledR}$ purchased from MCA (Winterswijk, The Netherlands). They consist of lyophilized human urine containing analytes specifically selected for laboratories active in the field of IEM. ORG ${ }^{\circledR}$ is intended for internal quality control of analytical systems for the determination of organic acids and acylglycines in the urine. The product was reconstituted with $10 \mathrm{~mL}$ of distilled water according to producer instructions.

\section{Chemometrics}

In this study, we used principal component analysis (PCA) and multiple linear regression (MLR) as chemometric tools to elaborate and interpret the results of the DoE.

PCA is a statistical procedure that analyzes a data table in which the results are described by inter-correlated quantitative dependent variables [19].

In this statistical procedure, an orthogonal transformation is used to convert the set of observations of possibly correlated variables (in our case, the peak areas of organic acids and acylglycines) into a set of values of linearly uncorrelated variables, i.e., the principal components. Each one of them can be represented as a vector and is a linear combination of the initial variables. Subsequently, the first principal component (called PC1) has the largest possible variance, because it accounts for as much of the variability in the data as possible.

This method is probably the best known and most widely used dimension-reducing technique, and it is very often applied in chemometric analysis, since it allows for eliminating the possible correlation among the original variables [19]. The number of principal components, at first, is the same of the original variables, but with a procedure of selection it is possible to use only the first two or three principal components, the ones that explain most part of the variability.

After the PCA, we have used an MLR model to interpret the results. MLR is one of the possible statistical models which can be used in regression analysis. In regression analysis, the focus is on the relationship between a dependent variable, also called the response variable, and one or more independent variables, called predictors [20]. In particular, an MLR model describes how a single response variable depends linearly on a number of predictor variables [21].

It shows the relationships between two or more predictor variables $(x)$ and one response variable (y), by fitting a linear equation (Equation (1)) to observed data.

$$
y=\beta_{0}+\beta_{1 \times 1}+\beta_{2 \times 2}+\ldots+\beta_{p \times p}+\varepsilon
$$


This equation can be also called the population regression line for $\mathrm{p}$ predictor variables: $\mathrm{x}_{1}, \mathrm{x}_{2}$, $\ldots x_{p} . \beta_{0}$ is the intercept of the line, while $\beta_{1}, \beta_{2}, \ldots \beta_{p}$ are the regression coefficients and $\varepsilon$ is the residual noise.

\section{Design of Experiment (DoE)}

In this study, the target of the DoE was to evaluate the effect of quantitative factors on the yield of organic acids to optimize the method used in the laboratory. The DoE consisted in a full-factorial design with four factors and two levels for each factor. The chosen factors were: the $\mathrm{pH}$ of the oximation reaction (A), the volume of $1 \mathrm{M}$ hydroxylamine solution (B), the GC injector temperature (C) and the volume of BSTFA $+1 \%$ of TMCS added for derivatization (D). These four factors were chosen on the basis of a comparison among our analytical method and two other methods: the one in use in Mayo Clinic Laboratories (Rochester, MN, USA) [22], and the one reported in the guidelines of American College of Medical Genetics and Genomic [23]. Two levels, coded with -1 and +1 , were selected for each factor (Table 1). The levels were chosen to comprehend the value actually applied in our analytical method and the values used by the other methods.

Table 1. Selected factors and their levels for design of experiment (DoE).

\begin{tabular}{cc}
\hline Factors & Levels: Values and Codes \\
\hline A: $\mathrm{pH}$ of oximation & $-1=7 ;+1=14$ \\
B: $\mathrm{V}_{\mathrm{NH} 2 \mathrm{OH}}(\mu \mathrm{L})$ & $-1=100 ;+1=500$ \\
C: $\mathrm{T}_{\text {inj }}\left({ }^{\circ} \mathrm{C}\right)$ & $-1=250 ;+1=285$ \\
D: $\mathrm{V}_{\text {BSTFA }}(\mu \mathrm{L})$ & $-1=50 ;+1=100$ \\
\hline
\end{tabular}

We have chosen to investigate the oximation step the most, because it is critical for the analysis of ketoacids, which are very important in the diagnosis of many organic acidurias. We have taken into account a range of $\mathrm{pH}$ between 7 and 14, because a basic $\mathrm{pH}$ should favor the reaction between organic ketoacids and hydroxylamine. The same reason is valid for the investigation of the volume of BSTFA $+1 \%$ of TMCS, used in the second derivatization. The temperature of the injection was considered because, if too high, it could cause a degradation of the derivatized organic acids and acylglycines. The response was considered to be the area of the chromatographic peak of each organic acid. In particular, we have selected 22 organic acids among the most important for the diagnosis of organic acidurias. The selected organic acids, including the internal standard, are reported below in Table 2, together with their retention times.

Table 2. List of organic acids and acylglycines considered for this study, with their respective retention times. As for the response area please see Table S1 in supplementary materials.

\begin{tabular}{cc}
\hline Analytes & Retention Times (min) \\
\hline 3-hydroxyisovaleric acid & 21.3 \\
4-hydroxybutiric acid & 22.9 \\
Methylmalonic acid & 23.4 \\
Ethylmalonic acid & 24.3 \\
Fumaric acid & 25.4 \\
Mevalonolactone & 26.9 \\
Glutaric acid & 27.3 \\
3-methylglutaric acid & 28.1 \\
3-methylglutaconic acid (2 peaks) & $28.6-31.2$ \\
Adipic acid & 31.4 \\
Pyroglutamic acid & 32.1 \\
2-hydroxyglutaric acid & 33.7 \\
Tiglylglycine & 33.9 \\
Tropic acid (internal standard) & 34.2 \\
3-hydroxy-3-methylglutaric acid & 34.5 \\
\hline
\end{tabular}


Table 2. Cont.

\begin{tabular}{cc}
\hline Analytes & Retention Times (min) \\
\hline Ketoglutaric acid & 35.4 \\
Hexanoylglycine & 36.1 \\
N-acetylspartic acid & 36.5 \\
Suberic acid & 37.7 \\
3-methylcitric acid (2 peaks) & $39.7-39.9$ \\
Sebacic acid & 40.4 \\
Vanilactic acid & 41.2 \\
\hline
\end{tabular}

Figure 1 shows an example of a chromatogram, obtained with this analysis, of the control material $\mathrm{ORG}^{\circledR}$. The identification and the integration of each peak is done automatically by the software. The most significant peaks are shown in Figure 1.

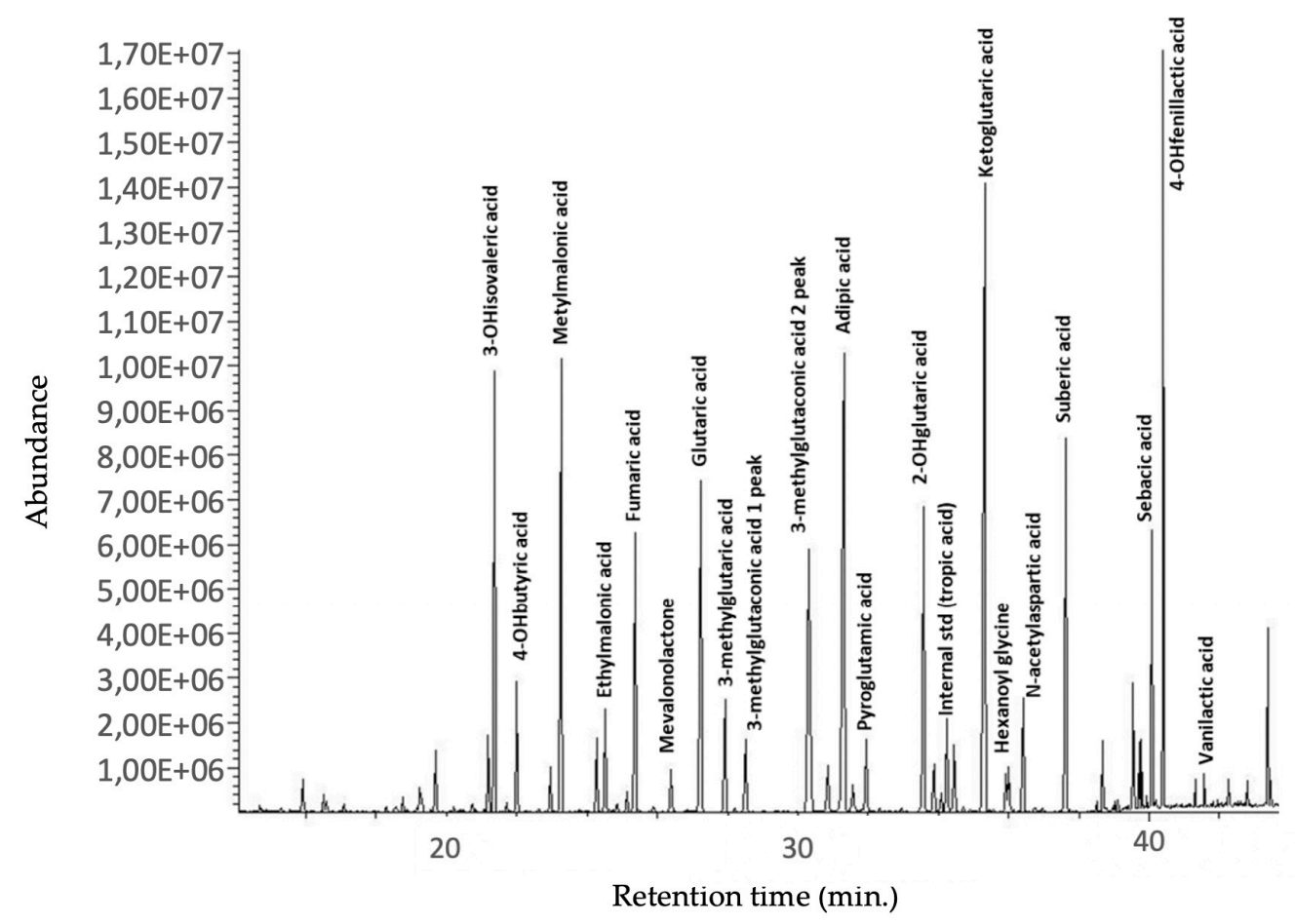

Figure 1. Example of an $O R G^{\circledR}$ chromatogram with the most significant organic acids and acylglycines. For a pathological patient chromatogram see Figure S3 in supplementary materials.

Software

All statistical analyses and interpretations were conducted using the software R [24], version 3.6.0. In particular, it was used the package CAT (Chemometric Agile Tool) developed by the Italian Group of Chemometrics of the SCI (Società Chimica Italiana, Roma, Italy) [25], freely available.

\section{Results and Discussion}

\section{Design of Experiment}

The multifactorial DoE was planned by comparing our analytical method with (i) procedures used in the most important IEM laboratory worldwide, Mayo Clinic's (Rochester, MN, USA), described by P. Rinaldo in his "Organic acids" [24], and (ii) those described in American College of Medical Genetics Guidelines [25]. In both reference procedures, the oximation of ketoacids is performed at a neutral $\mathrm{pH}$ and the temperature of injection in GC-MS is less than $250^{\circ} \mathrm{C}$, while, in our laboratory, 
the oximation $\mathrm{pH}$ is basic and the derivatized samples are injected at $285^{\circ} \mathrm{C}$. In the Mayo Clinic Laboratory, oximation is obtained with a volume of the reagent of $500 \mu \mathrm{L}$ versus $100 \mu \mathrm{L}$, and silanization is accomplished with an amount of BSTFA $+1 \%$ of TMCS of $100 \mu \mathrm{L}$ versus $50 \mu \mathrm{L}$, in comparison with our method. We developed a full-factorial design with four factors (k), i.e., $\mathrm{pH}$ and volume of the reagent of oximation, injection temperature and volume of silanizating agent, and two levels (L) for each factor. A total of 16 experiments $\left(\mathrm{L}^{\mathrm{k}}\right)$ were performed, as detailed in Table 3.

Table 3. Matrix of the DoE (Design of Experiments).

\begin{tabular}{ccccc}
\hline Experiment nr: & Factor $\mathbf{A}(\mathbf{p H})$ & $\begin{array}{c}\text { Factor } \mathbf{B} \\
\left(\mathbf{V}_{\mathbf{N H} \mathbf{2} \mathbf{H}}\right)\end{array}$ & Factor $\mathbf{C}\left(\mathbf{T}_{\mathbf{i n j}}\right)$ & Factor $\mathbf{D}\left(\mathbf{V}_{\mathbf{B S T F A}}\right)$ \\
\hline 1 & -1 & -1 & -1 & -1 \\
2 & +1 & -1 & -1 & -1 \\
3 & -1 & +1 & -1 & -1 \\
4 & -1 & -1 & +1 & -1 \\
5 & -1 & -1 & -1 & +1 \\
6 & +1 & +1 & -1 & -1 \\
7 & -1 & +1 & +1 & -1 \\
8 & -1 & -1 & +1 & +1 \\
9 & +1 & -1 & +1 & -1 \\
10 & -1 & +1 & -1 & +1 \\
11 & +1 & +1 & +1 & -1 \\
12 & -1 & +1 & +1 & +1 \\
13 & +1 & -1 & -1 & +1 \\
14 & +1 & -1 & +1 & +1 \\
15 & +1 & +1 & -1 & +1 \\
16 & +1 & +1 & +1 & +1 \\
\hline
\end{tabular}

The experiments were performed in random order without replicates. Randomization was necessary to guarantee the repeatability and to avoid any systematic error. According to the matrix of the DoE, the experiment number 9 corresponds to the method currently used in our laboratory.

Preliminary analysis of the results shows that there is not a positive trend for every factor: the variation of the factors from level -1 to level +1 not always leads to an increase in the response. In fact, the increase of factor $\mathrm{A}$, i.e., the $\mathrm{pH}$ of the oximation, raised the areas of the chromatographic peaks of some organic acids, while the increase of factors B and D, i.e., the volume of hydroxylamine solution and of BSTFA $+1 \%$ of TMCS, decreased the response for almost all the organic acids. The experiments that seemed to have the best result are number 9 and number 2. Experiment number 2 differs from number 9 only for the level of factor C.

Afterward, the autoscaling procedure was applied to the results, to give them all the same weight, and then the principal component analysis (PCA). The most significant principal components were considered to be the first and the second one (PC1 and PC2), since, together, they explain $53 \%$ of the variance (33\% and 19\% respectively). The scores plot and the loadings plot of the PCA are reported in Figure 2.

In the scores plot of the first two PCs, it is possible to notice that the experiment that maximizes both of the PCs is number 9, while the second-best experiment is number 2. They are circled in red in the Figure 2. The worst experiments are number 3, 10 and 7, with reference to the PCA.

The loadings plot shows that the direction in which most of the original variables, i.e., the organic acids, are maximized is the direction where PC1 is maximized.

To interpret the data, it is convenient to apply a MLR model with the following formula:

$$
y=b_{0}+b_{A} A+b_{B} B+b_{C} C+b_{D} D+b_{A B} A B+b_{A C} A C+b_{A D} A D+b_{B C} B C+b_{B D} B D+b_{C D} C D+\varepsilon
$$

where $y$ is the final response, $\varepsilon$ represents the noise and $b_{i}$ are the coefficients of each factor. The quadratic terms of all factors, which represent their reciprocal interactions, were also evaluated. With the MLR 
model, it was possible to obtain the coefficients plot for the factors and their binary interactions (Figure 3).
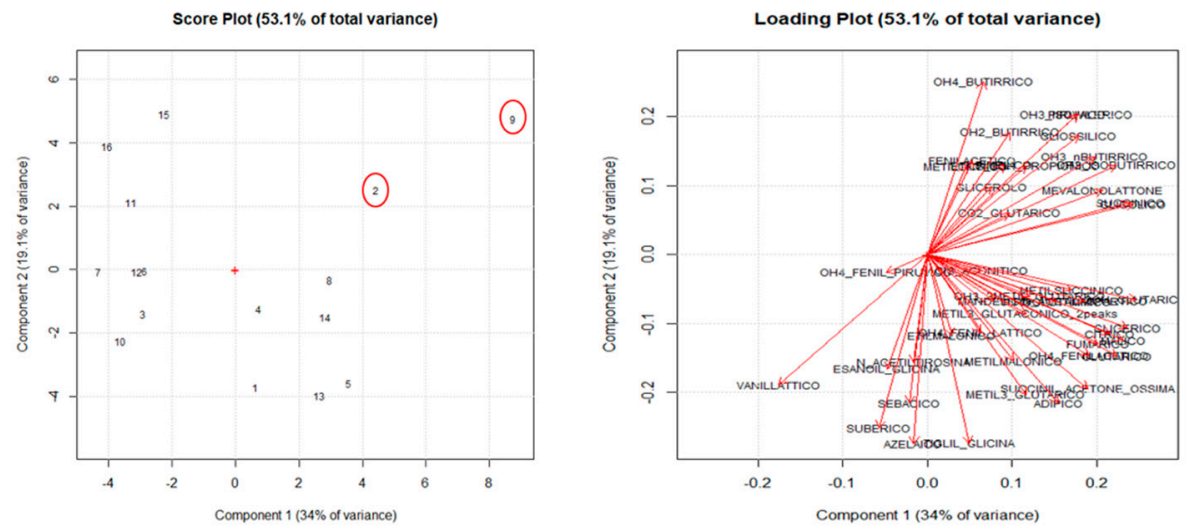

Figure 2. Scores plot of the first two principal components with the best experiments circled in red (left). Loadings plot of the first two principal components (right).

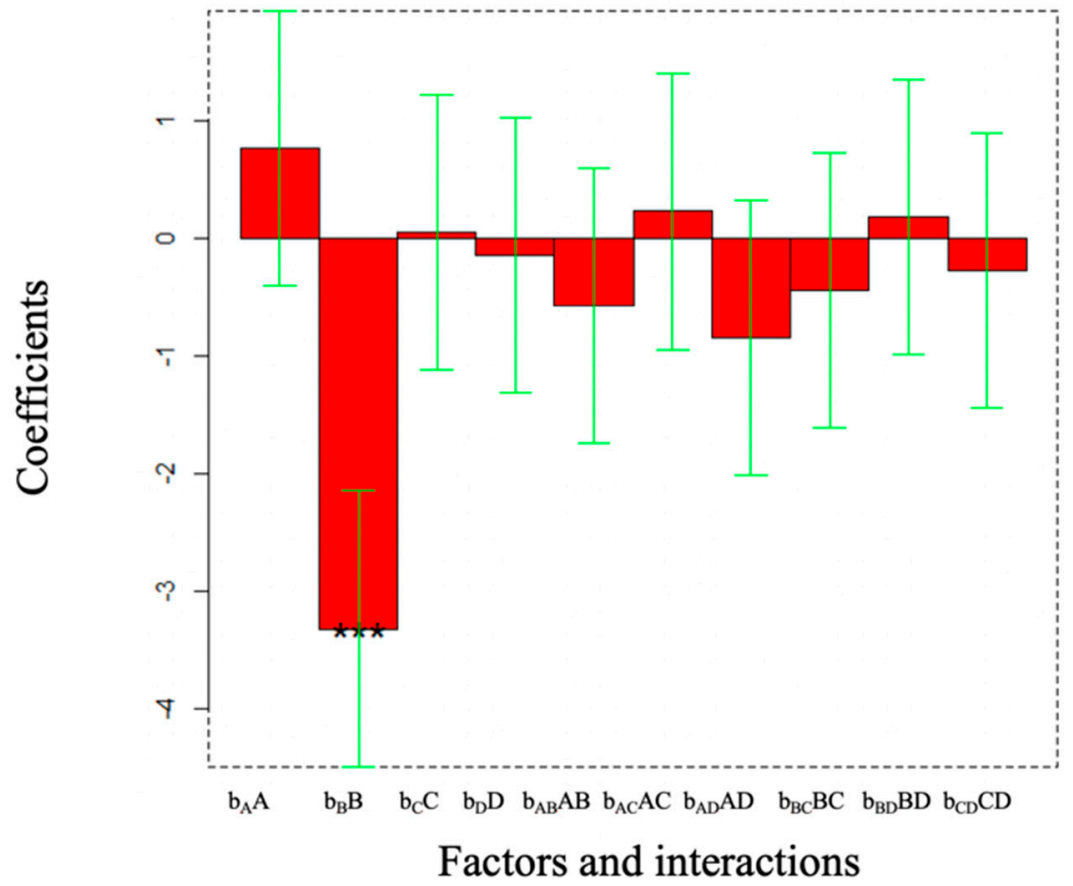

Figure 3. Coefficients plot for single factors $\left(b_{A} A, b_{B} B, b_{C} C, b_{D} D\right)$ and for their binary interactions $\left(b_{A B} A B, b{ }_{A C} A C, b_{A D} A D, b_{B C} B C, b_{B D} B D, b_{C D} C D\right)$. Significance level is indicated in the plot according to the following convention: ${ }^{* *}=p$-value $<0.001$.

The coefficients plot in Figure 3 shows that the most significant factor that affects the yield of organic acids is factor $B$, the volume of hydroxylamine solution, with a $p$-value $<0.001$. In this case, the analytical yield decreases when factor B increases from level -1 to level +1 . In all the other cases, the $p$-value results to be $>0.05$, indicating that the other factors and their interactions do not have a significant effect on the yield of organic acids.

The maximum response value for the DoE can be observed in the lower-right section of the response surface graphics (Figure 4), at the points encoded as $[+1,-1]$. We can conclude that the highest yield for organic acids was reached when the oximation $\mathrm{pH}$ was 14 , the volume of hydroxylamine solution was $100 \mu \mathrm{L}$, the GC injection temperature was $285^{\circ} \mathrm{C}$, and the volume of BSTFA $+1 \%$ of TMCS was $50 \mu \mathrm{L}$. These are exactly the experimental conditions used in our method. 

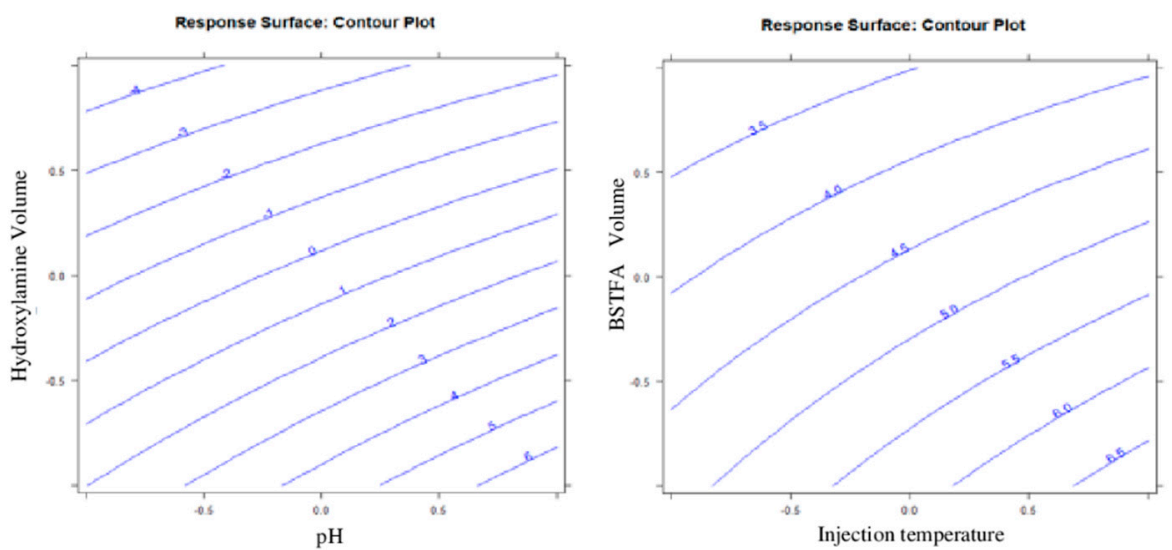

Figure 4. Contour plot of the full-factorial DoE: 2-D response surface plots for the first two factors of the DoE (Left) and for the second two factors (Right). The highest responses were observed: at the $\mathrm{pH}$ encoded as +1 with the volume of hydroxylamine solution encoded as -1 (right plot), and at the injection temperature encoded as +1 with the volume of $\mathrm{N}, \mathrm{O}$-Bis(trimethylsilyl)trifluoroacetamide (BSTFA) + $1 \%$ of trimethylchlorosilane (TMCS) encoded as -1 (left plot). Figures S1 and S2 of Supplementary Materials show the 3-D plots.

\section{Conclusions}

The present study has concluded, with a detailed investigation of the experimental parameters, that the method currently used in the laboratory is the most suitable for this analysis.

To obtain the highest yields in quantification of urinary organic acids the following conditions are recommended: basic $\mathrm{pH}$ and, a volume of $1 \mathrm{M}$ hydroxylamine solution not exceeding 100-150 $\mu \mathrm{L}$ for the oximation, $50 \mu \mathrm{L}$ of BSTFA $+1 \%$ TMCS for the sylanization and a temperature of $285^{\circ} \mathrm{C}$ for the injection of the derivatives in the GC.

The DoE has required accurate planning of the experiments and the use of statistical methods, such as PCA and MLR, necessary for the elaboration of the results. The major achievement of the present study is the demonstration that several experimental parameters can influence the results of the analysis of organic acids in urine. The results have shown some significant differences among the experiments.

Achieving optimal conditions for organic acids analysis represents, therefore, an important goal in order to obtain stable and reliable analytical results. In the future, a significant effort could be made to plan another DoE changing the levels of the factors, with the aim to define the optimal values for each factor. This statistical analysis confirmed that the method currently used in our laboratory was the best for the diagnosis of organic acidurias.

Supplementary Materials: The following are available online at http://www.mdpi.com/2673-4532/1/1/3/s1, Figure S1: Contour plot of the full-factorial DoE: 2-D response surface plots for the first two factors of the DoE, Figure S2: Contour plot of the full-factorial DoE: 3-D response surface plots for the second two factors, Figure S3: Example of a chromatogram obtained in case of a pathological patient, Table S1: Analyte name and response (peak area) for each experiment.

Author Contributions: Conceptualization: E.A., S.C., M.P.P., C.M.; methodology E.A., S.C., M.P.P.; software, E.A.; formal analysis, S.C., M.P.P.; resources, M.P.P.; data curation, M.P.; writing—original draft preparation, S.C., M.P.P.; writing-review and editing, M.P., S.C., E.A., M.P.P.; supervision: C.M., G.M. All authors have read and agreed to the published version of the manuscript.

Funding: This research received no external funding.

Conflicts of Interest: The authors declare no conflict of interest.

\section{References}

1. Solomons, T.W.G.; Fryhle, C.B.; Snyder, S.A. Organic Chemistry, 12th ed.; Wiley: Hoboken, NJ, USA, 2016. 
2. Mak, C.M.; Lee, H.-C.H.; Chan, A.Y.-W.; Lam, C.-W. Inborn errors of metabolism and expanded newborn screening, review and update. Crit. Rev. Clin. Lab. Sci. 2013, 50, 142-162. [CrossRef] [PubMed]

3. Villani, G.R.; Gallo, G.; Scolamiero, E.; Salvatore, F.; Ruoppolo, M. Classical organic acidurias: Diagnosis and pathogenesis. Clin. Exp. Med. 2017, 17, 305-323. [CrossRef] [PubMed]

4. Lehotay, D.C.; Clarke, J.T. Organic acidurias and related abnormalities. Crit. Rev. Clin. Lab. Sci. 1995, 32, 377-429. [CrossRef] [PubMed]

5. Tanaka, K.; Isselbacher, K.J. The Isolation and Identification of N-Isovalerylglycine from Urine of Patients with Isovaleric Acidemia. J. Biol. Chem. 1967, 242, 2966-2972. Available online: http://www.ncbi.nlm.nih. gov/pubmed/6027258 (accessed on 30 July 2020). [PubMed]

6. Christou, C.; Gika, H.G.; Raikos, N.; Theodoridis, G. GC-MS analysis of organic acids in human urine in clinical settings: A study of derivatization and other analytical parameters. J. Chromatogr. B 2014, 964, 195-201. [CrossRef] [PubMed]

7. Società Italiana Studio Malattie Metaboliche Ereditarie. Società Italiana Screenings Neonatali, Linee Guida per lo Screening Neonatale Esteso e la Conferma Diagnostica; SISMME, SISN: Genova, Italy, 2008.

8. Dietzen, D.J.; Rinaldo, P.; Whitley, R.J.; Rhead, W.J.; Hannon, W.H.; Garg, U.C.; Lo, S.F.; Bennett, M.J. National academy of clinical biochemistry laboratory medicine practice guidelines: Follow-up testing for metabolic disease identified by expanded newborn screening using tandem mass spectrometry: Executive summary. Clin. Chem. 2009, 55, 1615-1626. [CrossRef]

9. Tůma, P.; Samcová, E.; Štulík, K. Determination of the spectrum of low molecular mass organic acids in urine by capillary electrophoresis with contactless conductivity and ultraviolet photometric detection-An efficient tool for monitoring of inborn metabolic disorders. Anal. Chim. Acta 2011, 685, 84-90. [CrossRef] [PubMed]

10. Wang, S.-P.; Liao, C.-S. Comparison of ion-pair chromatography and capillary zone electrophoresis for the assay of organic acids as markers of abnormal metabolism. J. Chromatogr. A 2004, 1051, 213-219. Available online: http://www.ncbi.nlm.nih.gov/pubmed/15532576 (accessed on 30 July 2020). [CrossRef]

11. Rashed, M.S.; Ozand, P.T.; Harrison, M.E.; Watkins, P.J.F.; Evans, S.; Baillie, T.A. Electrospray Tandem Mass Spectrometry in the Diagnosis of Organic Acidemias, in Rapid Communication in Mass Spectrometry, 1st ed.; Wiley: Hoboken, NJ, USA, 1994; Volume 8, pp. 129-133. [CrossRef]

12. Pan, Z.; Gu, H.; Talaty, N.; Chen, H.; Shanaiah, N.; Hainline, B.E.; Cooks, R.G.; Raftery, D. Principal component analysis of urine metabolites detected by NMR and DESI-MS in patients with inborn errors of metabolism. Anal. Bioanal. Chem. 2006, 387, 539-549. [CrossRef] [PubMed]

13. Ombrone, D.; Salvatore, F.; Ruoppolo, M. Quantitative liquid chromatography coupled with tandem mass spectrometry analysis of urinary acylglycines: Application to the diagnosis of inborn errors of metabolism. Anal. Biochem. 2011, 417, 122-128. [CrossRef] [PubMed]

14. Nakagawa, K.; Kawana, S.; Hasegawa, Y.; Yamaguchi, S. Simplified method for the chemical diagnosis of organic aciduria using GC/MS. J. Chromatogr. B 2010, 878, 942-948. [CrossRef] [PubMed]

15. Burtis, C.; Ashwood, E.; Bruns, T.D. Textbook of Clinical Chemistry and Molecular Diagnostics, 5th ed.; Elsevier: Amsterdam, The Netherlands, 2012.

16. Jones, P.M.; Bennett, M.J. Urine Organic Acid Analysis for Inherited Metabolic Disease Using Gas Chromatography-Mass Spectrometry. Methods Mol. Biol. Hum. Press 2010, 603, 423-431. [CrossRef]

17. Hibbert, D.B. Experimental design in chromatography: A tutorial review. J. Chromatogr. B 2012, 910, $2-13$. [CrossRef]

18. Massart, D.L.; Vandeginste, B.G.M.; Buydens, L.M.C.; De Jong, S.; Lewi, P.J.; Smeyers-Verbeke, J. Handbook of Chemometrics and Qualimetrics: Part A, 1st ed.; Elsevier: Amsterdam, The Netherlands, 1997.

19. Bro, R.; Smilde, A.K. Principal component analysis. Anal. Methods 2014, 6, 2812-2831. [CrossRef]

20. Leardi, R. Experimental design in chemistry: A tutorial. Anal. Chim. Acta 2009, 652, 161-172. [CrossRef] [PubMed]

21. Draper, N.R.; Smith, H. Applied Regression Analysis (Electronic Source), Wiley Series in Probability and Statistics, 3rd ed.; Wiley: Hoboken, NJ, USA, 2014.

22. Rinaldo, P. Organic Acids in Laboratory Guide to the Methods in Biochemical Genetics, 1st ed.; Springer: Berlin, Germany, 2008; pp. 137-169.

23. Watson, M.S.; Lloyd-Puryear, M.A.; Mann, M.Y. American College of Medical Genetics (ACMG). Newborn Screening Expert Group-Main Report. Genet. Med. 2006, 117, S296-S307. 
24. R Core Team. R: A Language and Environment for Statistical Computing 2015. Available online: https: //www.r-project.org/ (accessed on 30 July 2020).

25. Gruppo di Chemiometria della Divisione di Chimica Analitica della Società Chimica Italiana (SCI), Chemometric Agile Tool (CAT). Available online: http://gruppochemiometria.it/index.php/software (accessed on 30 July 2020). 